Article

\title{
Antimicrobial Plant Extracts Encapsulated into Polymeric Beads for Potential Application on the Skin
}

\author{
Patrícia Rijo $^{1,2, *}$, Diogo Matias ${ }^{1}$, Ana S. Fernandes ${ }^{1,2}$, M. Fátima Simões ${ }^{2}$, Marisa Nicolai ${ }^{1}$ \\ and Catarina Pinto Reis ${ }^{1}$
}

1 CBiOS - Center for Research in Biosciences \& Health Technologies, Universidade Lusófona de Humanidades e Tecnologias, Campo Grande 376, Lisboa 1749-024, Portugal;

E-Mails: diogohcmatias@gmail.com (D.M.); ana.fernandes@ulusofona.pt (A.S.F.); mhfnicolai@gmail.com (M.N.); catarinapintoreis@gmail.com (C.P.R.)

2 Research Institute for Medicines and Pharmaceutical Sciences (iMed.UL), Faculdade de Farmácia da Universidade de Lisboa, Av. Prof. Gama Pinto, Lisboa 1649-003, Portugal;

E-Mail: fatimaalfaiatesimoes@gmail.com

* Author to whom correspondence should be addressed; E-Mail: patricia.rijo@ulusofona.pt; Tel.: +351-21-7515577/50; Fax: +351-21-7515598/79.

Received: 12 December 2013; in revised form: 4 February 2014 / Accepted: 10 February 2014 / Published: 18 February 2014

\begin{abstract}
In this study, the in vitro bacterial growth inhibition, antioxidant activity and the content in bioactive components of Plectranthus barbatus, $P$. hadiensis var. tomentosus, $P$. madagascarensis, $P$. neochilus and $P$. verticillatus aqueous extracts were investigated and compared by three extraction methods (infusion, decoction and microwave extractions). The microwave extract of $P$. madagascariensis showed the higher antimicrobial activity against the Staphylococcus epidermidis strain with a minimum inhibitory concentration of $40 \mu \mathrm{g} / \mathrm{mL}$. This extract also showed no toxicity in a general toxicity assay and no considerable cytotoxicity against a human keratinocyte cell line. The antioxidant activity of the extracts was assessed using 2,2-diphenyl-1-picrylhydrazyl (DPPH method), and all showed antioxidant activity. The microwave extract of $P$. madagascariensis was the one with the highest antioxidant activity ( $I C_{50}$ value of $41.66 \mu \mathrm{g} / \mathrm{mL}$ ). To increase extract stability, the microwave $P$. madagascariensis extract was then successfully encapsulated into alginate beads with high efficiency. This effective and low-cost strategy seems to be easy to extrapolate to an industrial scale with a future application on the skin.
\end{abstract}


Keywords: Plectranthus; antimicrobial activity; antioxidant activity; polymeric beads; alginate; extraction methods

\section{Introduction}

Natural products, widely used in traditional medicine, are a common source of bioactive molecules for the treatment of bacterial infections. Particularly, plants of the Plectranthus genus (Lamiaceae) have several ethnobotanical documented uses, including the treatment of various infections [1,2]. These traditional uses are well supported by the isolation of diverse antimicrobial metabolites, namely diterpenoids and polyphenols [3-5]. The use of herbal extracts as therapeutic agents may be an interesting area for developing countries and has aroused the interest of the population in developed countries. However, aqueous extracts generally present some formulation problems, such as long-term instability, low bioavailability and a significant burst release.

Biodegradable and biocompatible polymers can be used as carriers of pharmaceutical active ingredients and are a useful strategy for the enhancement of their stability characteristics. Alginate is a low-cost and biodegradable polymer. "Alginate" is the term typically used for the salts of alginic acid, but it can also refer to all the derivatives of alginic acid and alginic acid itself. Alginic acid is an algal polysaccharide and a species of polycarboxylic acid. It consists of $\alpha$-L-guluronic acid (G) and $\beta$-D-mannuronic acid $(\mathrm{M})$, and their relative proportion $(\mathrm{M} / \mathrm{G})$ ratio determines the biofunctional properties of alginic acid [6]. The gelling properties of alginate depend strongly upon its monomeric composition, sequential arrangements and the lengths of the G-blocks [7]. Nowadays, alginates are widely used in food, textile industry and other fields, including paper coating, pharmaceuticals and welding rods. In the pharmaceutical industry, alginates are generally applied as the thickening, gelling, stabilizing, suspending, controlled release and emulsifying agents [8]. Products originated by this way can have several applications, such as drug delivery [9,10]. Different techniques could be used in the encapsulation process, such as internal and external gelations [8], ultrasonic assisted atomization [11] and the spray drying process [12]. In some encapsulation methods, the active ingredient is encapsulated into calcium alginate beads and slowly released from the polymer matrix when the bead is exposed in the appropriate environment $[13,14]$. In general, alginate solutions are stable in the pH range 5.5-10 at room temperature for a long time, and one approach to improve the calcium alginate bead stability may be obtained with the medium enrichment with calcium ions, where the beads are suspended [15].

\section{Experimental Section}

\subsection{Chemicals}

Alginic acid sodium salt from brown algae [low glucuronic content (named as Alginate Low G) with low viscosity (4-12 cPs) $1 \%$ in $\mathrm{H}_{2} \mathrm{O}$ at $25{ }^{\circ} \mathrm{C}$, dimethyl sulphoxide (DMSO), 2,2-diphenyl-1-picrylhydrazyl (DPPH), 2,6-di-tert-butyl-4-hydroxytoluene (BHT), chlorogenic and rosmarinic acids, phosphate buffered saline (PBS; $0.01 \mathrm{M}, \mathrm{pH}$ 7.4), trypsin, Dulbecco's Modified Eagle's Medium (DMEM), penicillin-streptomycin solution, fetal bovine serum and thiazolyl blue 
tetrazolium bromide (MTT) were purchased from Sigma-Aldrich (Steinheim, Germany). All chemicals were of analytical grade.

\subsection{Plant Material}

The whole plant material of Plectranthus barbatus Andrews, P. hadiensis (Forssk.) Schweinf. ex Spreng. var. tomentosus (Benth.) Codd, P. madagascarensis (Lam.) Benth., P. neochilus Schltr. and P. verticillatus (L.F.). Druce grown in the Parque Botânico da Tapada da Ajuda from cuttings provided by the Kirstenbosch National Botanical Gardens, South Africa, were collected between 2007 and 2008, always in June and September, and voucher specimens were deposited in the Herbarium "João de Carvalho e Vasconcellos” of the “Instituto Superior de Agronomia”, Lisboa (LISI), Portugal.

\subsection{Extract Preparation}

Aqueous extracts of each plant were prepared using $10 \mathrm{mg}$ of dried and powdered plant material in $100 \mathrm{~mL}$ of distilled water and then filtered through Whatman N. 5 paper (Whatman, Inc., Clifton, NJ, USA.) by different extraction techniques: (1) decoction extracts were prepared by boiling the plant material in distilled water for $10 \mathrm{~min}$; (2) infusion extracts were prepared by adding freshly boiled distilled water to plant material for $10 \mathrm{~min}$; (3) microwave extracts were prepared by using the plant material in distilled water in a conventional microwave oven for $2 \mathrm{~min}$ at a continuous irradiation of $2.45 \mathrm{GHz}$. From every prepared extract, an aliquot of $1 \mathrm{~mL}$ was freeze-dried (Freezone $2.5 \mathrm{~L}$, Freeze-dryer Labconco, Kansas City, MO, USA) and the dry weight determined on the average of three independent measures (Table 1).

\subsection{High-Performance Liquid Chromatography (HPLC) Analysis}

The determination of the extracts' main components was done by HPLC analysis and was carried out in an Agilent Technologies 1200 Infinity Series LC System equipped with diode array detector (DAD), ChemStationSoftware and a LiChrospher $^{\circledR} 100$ RP-18 (5 mm) column from Merck (Darmstadt, Germany). Extracts were analyzed by injecting $20 \mu \mathrm{L}$ and using a gradient composed of Solution A (methanol), Solution B (acetonitrile) and Solution D (0.3\% trichloroacetic acid in water) as follows: 0 min, 15\% A, 5\% B and 80\% D; 20 min, 80\% A, 10\% B and 10\% D; 25 min, 80\% A, 10\% B and $10 \% \mathrm{D}$; and $28 \mathrm{~min}, 15 \% \mathrm{~A}, 5 \% \mathrm{~B}$ and $80 \% \mathrm{D}$. The flow rate was set at $1 \mathrm{~mL} / \mathrm{min}$. The standards were run under the same conditions in methanol, and the detection was carried out between 200 and $600 \mathrm{~nm}$ with a diode array detector (DAD). All analyses were performed in triplicate.

\subsection{Microorganisms and Well Diffusion Method}

The antimicrobial activity of each prepared extract was evaluated against six bacterial species obtained from the American Type Culture Collection (ATCC), namely Bacillus subtilis ATCC 6633, Enterococcus faecalis ATCC 29212, Escherichia coli ATCC 25922, Pseudomonas aeruginosa ATCC 27853, Staphylococcus aureus ATCC 25923 and S. epidermidis ATCC 12228. The well diffusion assay was used to screen the extracts with antimicrobial activity. 
Previously prepared extracts were freeze-dried using the same equipment as Section 2.3 and reconstituted in DMSO to a $1 \mathrm{mg} / \mathrm{mL}$ concentration. Stock solutions of reference antibiotics (vancomycin and norfloxacin) were also prepared to $1 \mathrm{mg} / \mathrm{mL}$ in DMSO.

In aseptic conditions, Petri dishes containing $20 \mathrm{~mL}$ of solid Mueller-Hinton culture medium were inoculated with $0.1 \mathrm{~mL}$ of bacterial suspension matching a 0.5 McFarland standard solution and uniformly spread on the medium surface using a sterile swab. Wells of approximately $5 \mathrm{~mm}$ in diameter were made in the medium with a sterile glass Pasteur pipette and $50 \mu \mathrm{L}$ of each extract were added into the well. A positive control of vancomycin for Gram-positive bacteria, norfloxacin for Gram-negative bacteria and a negative control of DMSO were used. Plates were incubated at $37^{\circ} \mathrm{C}$ for $24 \mathrm{~h}$. The antibacterial activity was evaluated by measuring the diameter ( $\mathrm{mm}$ ) of the inhibition zone formed around the wells and compared to controls.

\subsection{Determination of Minimum Inhibitory Concentrations (MICs)}

Antibacterial activity was also determined using the microplate broth microdilution method. In aseptic conditions, $100 \mu \mathrm{L}$ of liquid Mueller-Hilton medium was distributed in each well of a 96-well plate. To the first well of each row was added $100 \mu \mathrm{L}$ of extract, the positive control or negative control solutions at a $1 \mathrm{mg} / \mathrm{mL}$ concentration. Using a multichannel micropipette, a serial dilution was made to $1: 2$ proportion between each row of wells $(1.95-500 \mu \mathrm{g} / \mathrm{mL}$ range). Lastly, $10 \mu \mathrm{L}$ of bacterial suspension were added to every well and plates were covered and incubated at $37{ }^{\circ} \mathrm{C}$ for $24 \mathrm{~h}$. The bacterial growth was measured with an absorbance microplate reader (Thermo Scientific Multiskan FC, Loughborough, UK) set to $620 \mathrm{~nm}$. Assays were carried out in triplicate for each tested microorganism.

\subsection{Determination of Antioxidant Activity}

To evaluate the radical scavenging activity, the DPPH method was used [16]. Ten microliters of plant extract were added to a $990 \mu \mathrm{L}$ solution of $\mathrm{DPPH}(0.002 \%$ in methanol). The mixture was incubated for $30 \mathrm{~min}$ at room temperature. The absorbance was measured at $517 \mathrm{~nm}$ against a corresponding blank and the antioxidant activity was calculated as:

$$
A A \%=\frac{A_{\mathrm{DPPH}}-A_{\text {sample }}}{A_{\mathrm{DPPH}}} \times 100
$$

where $A A$ is the antioxidant activity, $A_{\mathrm{DPPH}}$ is the absorption of DPPH against the blank and $A_{\text {sample }}$ is the absorption of the extract or control against the blank. Tests were carried out in triplicate, and the sample concentration providing $50 \%$ of antioxidant activity $\left(I C_{50}\right)$ was obtained by plotting antioxidant activity against the sample concentration.

\subsection{Screening of General Toxicity}

To evaluate general eukaryotic toxicity, the Saccharomyces cerevisiae model developed by Roberto and Caetano was applied [17]. An inoculum of S. cerevisiae was added to $20 \mathrm{~mL}$ of YPD liquid medium (YPD means yeast extract $1 \%$, peptone $0.5 \%$ and dextrose $2 \%$ ) in a sterile Erlenmeyer 
flask and left to incubate for $16-24 \mathrm{~h}$ at $30{ }^{\circ} \mathrm{C}$ with 230 stirring cycles per min. After incubation, the absorbance of the yeast solution was measured in a UV-visible spectrophotometer (Thermo Scientific Evolution 600, Loughborough, UK) at $525 \mathrm{~nm}$ and diluted with YPD liquid medium to an approximated initial absorbance of 0.2 mUA [approximate $10^{7}$ colony forming unities (CFU) per mL]. Two milliliters of diluted yeast solution and $0.2 \mathrm{~mL}$ of sample or control were added to $4 \mathrm{~mL}$ plastic disposable cuvettes, and then, they were maintained at $30{ }^{\circ} \mathrm{C}$ with 230 stirring cycles per minute for $6 \mathrm{~h}$. At each 30-min interval, the yeast culture was resuspended for $3 \mathrm{~s}$ by vortex agitation and the absorbance at $525 \mathrm{~nm}$ was measured. The growth curve of each control and sample was made, and the slope of exponential phase of the growth determined. The inhibition of growth was determined as:

$$
G I \%=\frac{m_{\text {sample }}}{m_{\text {control }}} \times 100
$$

where $G I \%$ is the growth inhibition percent, $m_{\text {sample }}$ is the slope of the growth curve in the exponential phase of the treated yeast culture and $m_{\text {control }}$ is the slope of the growth curve in the exponential phase of the untreated yeast culture. Tests were carried out in triplicate.

\subsection{Evaluation of Cytotoxicity against the HaCaT Line (Human Adult Low-Calcium} High-Temperature Keratinocytes)

The cytotoxicity profile of the extracts was characterized in the human keratinocyte cell line, HaCaT. HaCat cells were cultured in DMEM supplemented with $10 \%$ fetal bovine serum, $100 \mathrm{U} / \mathrm{mL}$ penicillin and $0.1 \mathrm{mg} / \mathrm{mL}$ streptomycin. Cell viability was evaluated using a $24 \mathrm{~h}$-incubation protocol by the MTT assay, according to a procedure adapted from Fernandes et al. [18]. Two independent experiments were performed, and four replicate cultures were used in each independent experiment.

\subsection{Production of Plectranthus spp. Extract-Loaded Calcium Alginate Beads}

Calcium alginate beads were prepared by the extrusion/external gelation method [14]. A volume of $1 \mathrm{~mL}$ of reconstituted extract at $10 \mathrm{mg} / \mathrm{mL}$ was added to $5 \mathrm{~mL}$ of $2 \%$ sodium alginate solution. This suspension was extruded through a fine syringe (21 gauge, diameter $0.81 \mathrm{~mm}$ ) to $100 \mathrm{~mL}$ of $\mathrm{CaCl}_{2}$ $0.05 \mathrm{M}$ solution under magnetic stirring for $15 \mathrm{~min}$. The resulting solution was filtered to obtain gelled and hydrated beads.

\subsection{Characterization of Plectranthus spp. Extract-Loaded Calcium Alginate Beads}

The size and morphology of the calcium alginate beads were assessed by direct observation, and the images were recorded digitally. The encapsulation efficiency of Plectranthus spp. extract in calcium alginate beads was determined through an indirect method in triplicate. The supernatant obtained in the preparation of beads was injected directly in the HPLC-DAD using the method described previously. The rosmarinic acid concentration was used as the indicator of encapsulation efficiency using the following formula:

$$
E E \%=\frac{C_{\mathrm{i}}-C_{\mathrm{f}}}{C_{\mathrm{i}}} \times 100
$$


where $E E \%$ corresponds to the encapsulation efficiency, $C_{\mathrm{i}}$ to the initial concentration of rosmarinic acid and $C_{\mathrm{f}}$ to the final concentration of rosmarinic acid (non-encapsulated or free rosmarinic acid) present in the supernatant.

\subsection{Stability Studies of Plectranthus spp. Extract-Loaded Calcium Alginate Beads}

\subsubsection{Stability over the Time}

Hydrated and filtered beads were left at room temperature for 7 days and then liquefied in $50 \mathrm{~mL}$ of $55 \mathrm{mM}$ of sodium citrate. The amount of rosmarinic acid in the supernatant and in the remaining beads was quantified using the same HPLC method described before. Fresh extracts were used as the controls.

\subsubsection{Stability against UV Radiation}

Two hundred milligrams of freeze-dried calcium alginate beads were placed in vials containing $10 \mathrm{~mL}$ of water under UV light (274 nm) and left for magnetic stirring for $2 \mathrm{~h}$. The amount of rosmarinic acid in the supernatant and remaining in beads was quantified using the same HPLC method. Fresh extracts were used as the controls.

\section{Results and Discussion}

\subsection{Extraction Yields}

Aqueous extracts of $P$. barbatus, $P$. hadiensis var. tomentosus, $P$. madagascarensis, $P$. neochilus and $P$. verticillatus were prepared by three methods: decoction, infusion and microwave. Aliquots of the resulting extracts were freeze-dried to determine the dry weight of each extract (Table 1). The best yield was verified for the $P$. barbatus infusion extract with a mean value of $33 \pm 3.0 \mathrm{mg} / \mathrm{mL}$. It could also be identified that the trend of the infusion technique led to a higher dry weight and in the microwave extracts, the lowest dry weight, except for $P$. verticillatus, where only the microwave was determined.

Table 1. Aqueous extract dry weights for each Plectranthus species and extraction method.

\begin{tabular}{ccc}
\hline Plant & Extraction method & Weight of dry extract (mg/mL) \\
\hline \multirow{3}{*}{ P. barbatus } & Decoction & $26 \pm 2.0$ \\
& Infusion & $33 \pm 3.0$ \\
& Microwave & $22 \pm 1.0$ \\
\hline \multirow{3}{*}{ P. hadiensis var. Tomentosus } & Decoction & $32 \pm 2.0$ \\
& Infusion & $26 \pm 1.0$ \\
& Microwave & $17 \pm 1.0$ \\
\hline \multirow{2}{*}{ P. madagascarensis } & Decoction & $22 \pm 1.0$ \\
& Infusion & $26 \pm 4.0$ \\
& Microwave & $15 \pm 4.0$ \\
\hline \multirow{2}{*}{ P. neochilus } & Decoction & $22 \pm 2.0$ \\
& Infusion & $23 \pm 10.0$ \\
& Microwave & $11 \pm 4.0$ \\
\hline \multirow{2}{*}{ P. verticillatus } & Decoction & nd \\
& Infusion & nd \\
\hline
\end{tabular}

Note: nd, not determined. 


\subsection{Phytochemical Composition of Extracts}

The prepared Plectranthus spp. aqueous extracts were analyzed by HPLC-DAD and their main constituents identified by comparison of their UV absorbance spectra with standard UV absorbance spectra. Chlorogenic and rosmarinic acids were identified in all extracts (Table 2), and in $P$. madagascariensis extracts, the rosmarinic acid yield was quantified (Figure 1).

The calculated calibration curve for rosmarinic acid was $y=5175.9 x-127.46\left(R^{2}=0.999\right)$.

Table 2. Identified polyphenols and antioxidant activity of prepared aqueous Plectranthus extracts.

\begin{tabular}{cccc}
\hline Plant & Extraction method & $\begin{array}{c}\text { Identified polyphenols } \\
(\boldsymbol{w} / \boldsymbol{w} \% \text { of extract })\end{array}$ & Antioxidant activity (AA\%) \\
\hline \multirow{3}{*}{ P. barbatus } & Decoction & CA, RA & 10.30 \\
& Infusion & CA, RA & 15.70 \\
& Microwave & CA, RA & 10.10 \\
\hline \multirow{3}{*}{ P. hadiensis var. tomentosus } & Decoction & CA, RA & 17.9 \\
& Infusion & CA, RA & 40.0 \\
& Microwave & CA, RA & 32.6 \\
\hline \multirow{3}{*}{ P. madagascarensis } & Decoction & CA, RA (15.72) & 20.60 \\
& Infusion & CA, RA (10.93) & 10.20 \\
& Microwave & CA, RA (18.64) & 17.20 \\
\hline \multirow{3}{*}{ P. neochilus } & Decoction & CA, RA & 12.1 \\
& Infusion & CA, RA & 14.7 \\
\hline P. verticillatus & Microwave & CA, RA & 14.0 \\
\hline
\end{tabular}

Notes: CA, chlorogenic acid; RA, rosmarinic acid and w/w, weight/weight.

Figure 1. Example of a chromatogram of $P$. madagascariensis extract $(5 \mathrm{mg} / \mathrm{mL})$ obtained by the microwave technique. (a) Rosmarinic acid structure.

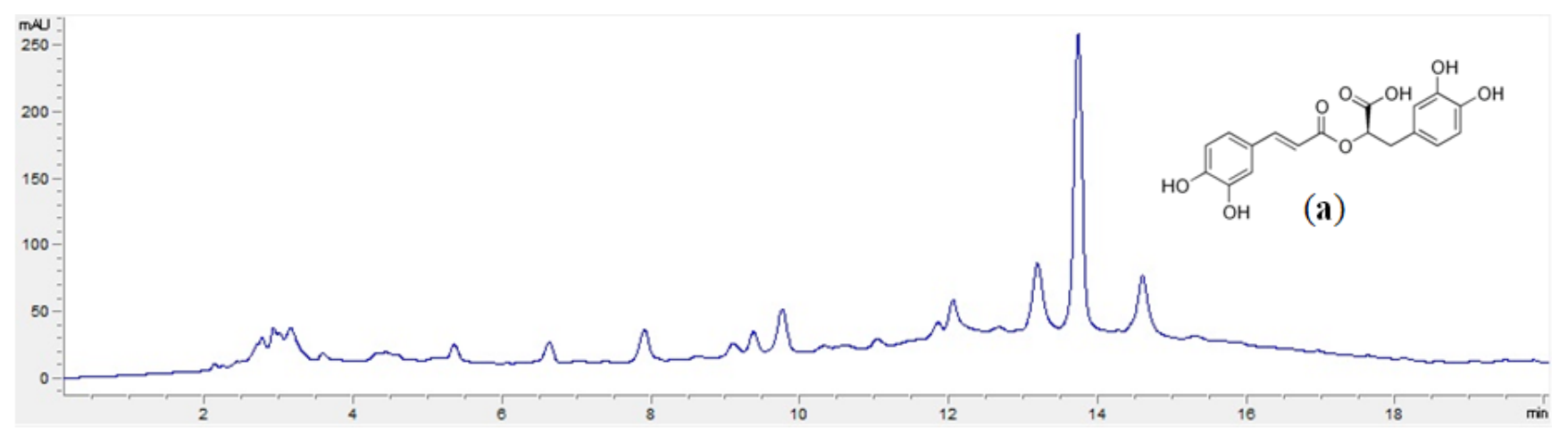

\subsection{Antibacterial Activity}

The antibacterial activities of the prepared aqueous Plectranthus extracts were screened initially by well diffusion assay, and only the $P$. madagascariensis microwave extract showed a $10 \mathrm{~mm}$ of inhibition zone against $S$. epidermidis. This extract showed a potent antimicrobial activity against S. epidermidis with a minimum inhibitory concentration (MIC) value of $40 \mu \mathrm{g} / \mathrm{mL}$. 


\subsection{Antioxidant Activity}

All extracts in this study showed antioxidant activity at $10 \mu \mathrm{g} / \mathrm{mL}$ (Table 2) and the P. madagascariensis extracts were the most active (10.2\% to $20.6 \%$ of antioxidant activity). Thus, the P. madagascariensis microwave extract was elected for further studies, considering the results obtained on antimicrobial activity (Section 3.3) and the $\mathrm{IC}_{50}$ of $41.66 \mu \mathrm{g} / \mathrm{mL}$.

\subsection{General Toxicity}

The $S$. cerevisiae model is a simple and effective model to predict general eukaryotic toxicity. This technique was applied to the P. madagascariensis extract and no toxicity was verified.

\subsection{Cytotoxicity}

The cytotoxicity profile of $P$. madagascariensis microwave extract was studied in a human keratinocyte cell line (HaCaT) using the MTT assay. For concentrations up to $500 \mu \mathrm{g} / \mathrm{mL}$ and an incubation period of $24 \mathrm{~h}$, no considerable cytotoxic effects were observed, as shown in Figure 2.

Figure 2. The effect of the $P$. madagascariensis extract on the viability of human keratinocytes. Results are the average values \pm SD of two independent experiments, each comprising four replicate cultures. MTT, thiazolyl blue tetrazolium bromide.

\section{P. madagascariensis - microwave extract}

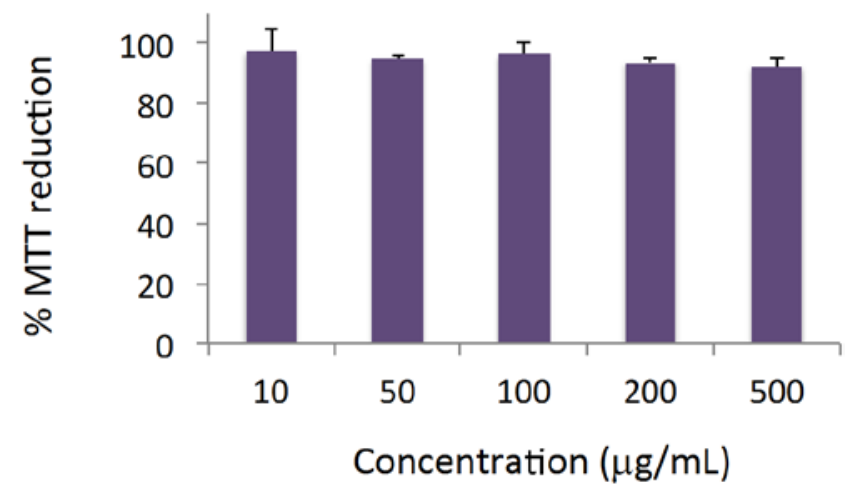

\subsection{Production and Characterization of Plectranthus spp. Extract-Loaded Calcium Alginate Beads}

Calcium alginate beads with herbal extracts were successfully prepared using the extrusion/external gelation method. This method was very rapid and can be easily adapted to the industrial scale. In general, there are two main techniques for producing calcium alginate beads, based on the source of the calcium. In external gelation method, alginate droplets are gelled in a calcium chloride solution, and thus, the calcium source is external to the droplet. Gelation is initiated from the surface and works toward the inner core. In contrast, the internal gelation method uses an internal source of calcium, where the alginate solution is preloaded within soluble calcium salt, releasing calcium ions with $\mathrm{pH}$ adjustment $[8,14]$. In this study, calcium alginate beads were spherical in shape and showed a mean diameter of $2.57 \pm 0.21$ and $2.33 \pm 0.19 \mathrm{~mm}$ for loaded and empty particles, respectively, in the digital images (Figure 3). These images confirmed the encapsulation of the herbal extracts inside the calcium 
alginate beads since empty calcium alginate beads were transparent; meanwhile, when the extract was presented, the beads changed to yellow.

Figure 3. Digital images of (a) the herbal extract-loaded calcium alginate beads and (b) the empty calcium alginate beads.

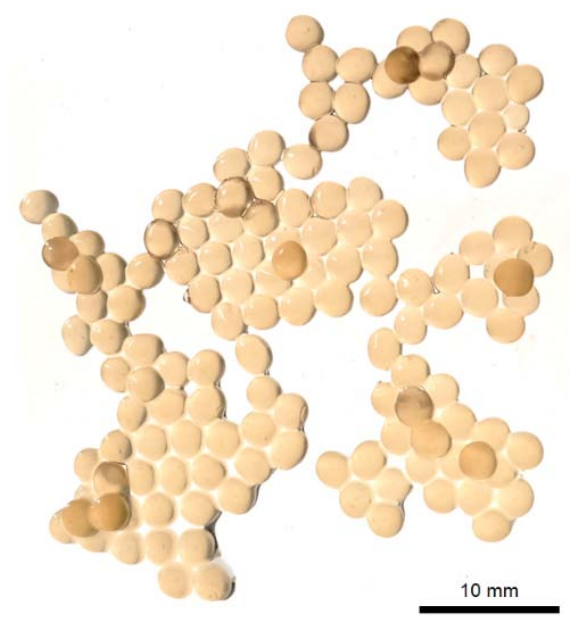

(a)

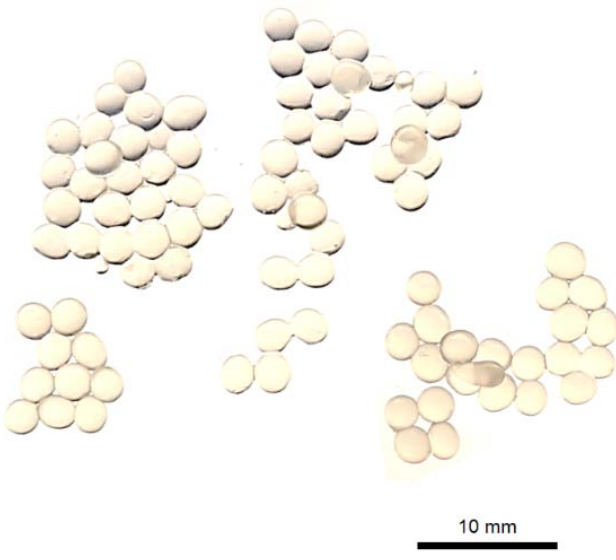

(b)

Alginate is a polyelectrolyte hydrocolloid, so it interacts with water, reducing its diffusion and stabilizing its presence. Such water may be held specifically through direct hydrogen bonding or the structuring of water within extensive, but contained, inter- and intra-molecular voids. In this study, an aqueous microwave $P$. madagascariensis extract was successfully encapsulated into calcium alginate beads, where the encapsulation efficiency value was very high (around 88\%). Alginate and the encapsulated extracts have the same hydrophilic properties, and thus, a high value of encapsulation efficiency was expected. The gelling properties of alginate strongly depend upon its monomeric composition, sequential arrangements and the lengths of the G-blocks [7]. As previously described, alginate forms gels with calcium, and this is an auto-cooperative reaction that occurs between calcium and the homopolymeric blocks of $\mathrm{G}$ on the alginate chain $[7,19]$. Calcium alginate gels shrink during gel formation. This leads to the loss of water and an increase in the concentration of polymer in the beads relative to that in the alginate solution and may be reducing some of the encapsulant diffusion to outside of the polymeric matrix. Herein, the physical characteristics of calcium alginate gel are also influenced by the alginate concentration and molecular size, the calcium concentration and gelling time [20]. In addition, in the extrusion/external gelation method, sodium alginate solution is forced into a bath of a calcium chloride solution, and then, calcium alginate is formed as fibers (gel). If low viscosity alginates are used, a strong solution is generally formed without any viscosity problems, and the calcium bath is not diluted as rapidly. In general, low G alginates, like we used in this study, produced very flexible gels [15]. Gel fibers have very good strength in both wet and dry forms, and as with most polymer fibers formed by extrusion, stretching while forming increases the linearity of the polymer chains and the strength of the fiber. 


\subsection{Stability Studies}

Microencapsulation is a very useful process utilized in the incorporation of active ingredients within polymers aiming at, among other objectives, the prolonged release of pharmaceutical compounds and protection from atmospheric agents (moisture, light, heat and/or oxidation) [21]. The extract contents of chlorogenic acid (CA) and rosmarinic acid (RA) were maintained in calcium alginate beads over time (for seven days) and against UV in contrast to free extracts (or non-encapsulated). Similarly to previous studies [8,22,23], alginate proved to be a very good encapsulant material. Future studies are now on-going and include the incorporation of this system into a topical dosage form and its potential application on the skin.

\section{Conclusions}

In this study, a $P$. madagascariensis aqueous microwave extract was identified as possessing relevant anti-S. epidermidis activity along with antioxidant capacity and low toxicity in the models tested. Rosmarinic acid was identified as the main component of this extract. These properties may be beneficial for the healing of a variety of skin conditions, this extract being a potential antibacterial skin active ingredient. To prevent the long-term instability of the extract, the encapsulation of the P. madagascariensis microwave extract into a polymeric matrix was successfully obtained. This encapsulation method produced several and spherical particles, homogenous in color and particle size and with very high encapsulation efficiency. The long-term stability studies also demonstrate the viability of this system to the improvement of the stability of this extract and its biological activities.

\section{Acknowledgments}

The authors are thankful to Amílcar Roberto (Universidade Lusófona de Humanidades e Tecnologias, Lisbon, Portugal) for providing the original yeast culture and culture materials.

\section{Conflicts of Interest}

The authors declare no conflict of interest.

\section{References}

1. Lukhoba, C.W.; Simmonds, M.S.J.; Paton, A.J. Plectranthus: A review of ethnobotanical uses. J. Ethnopharmacol. 2006, 103, 1-24.

2. Rice, L.J.; Brits, G.J.; Potgeiter, C.J.; van Staden, J. Plectranthus: A plant for the future? South Afr. J. Bot. 2011, 77, 947-959.

3. Rijo, P.; Gaspar-Marques, C.; Simões, M.F.; Duarte, A.; Apreda-Rojas, M.C.; Cano, F.H.; Rodriguez, B. Neoclerodane and labdane diterpenoids from Plectranthus ornatus. J. Nat. Prod. 2002, 65, 1387-1390.

4. Simões, M.F.; Rijo, P.; Duarte, A.; Barbosa, D.; Matias, D.; Delgado, J.; Cirilo, N.; Rodriguez, B. Two diterpenoids from Plectranthus species. Phytochem. Lett. 2010, 3, 221-225. 
5. Rijo, P.; Simões, M.F.; Francisco, A.P.; Rojas, R.; Gilman, R.H.; Vaisberg, A.J.; Rodriguez, B.; Moiteiro, C. Antimycobacterial metabolites from Plectranthus: Roylone derivates against mycobacterium tuberculosis strains. Chem. Biodivers. 2010, 7, 922-932.

6. Murata, Y.; Nakada, K.; Miyamoto, E.; Kawashima, S.; Seo, S.-H. Influence of erosion of alcium-induced alginete gel matrix on the release of Brilliant Blue. J. Control. Rel. 1993, 23, 21-26.

7. Martinsen, A.; Skjak Braek, G.; Smidsrod, O. Alginate as immobilization material: I. Correlation between chemical and physical properties of alginate beads. Biotechnol. Bioeng. 1989, 33, 79-89.

8. Reis, C.P.; Neufeld, R.J.; Vilela, S.; Ribeiro, A.J.; Veiga, F. Review and current status of emulsion/dispersion technology using an internal gelation process for the design of alginate particles. J. Microencapsul. 2006, 23, 245-257.

9. Liakos, I.; Rizzello, L.; Bayer, I.; Pompa, P.; Cingolani, R.; Athanassiou, A. Controlled antiseptic release by alginate polymer films and beads. Carbohydr. Polym. 2013, 92, 176-183.

10. Liakos, I.; Rizzello, L.; Scurr, D.; Pompa, P.; Bayer, I.; Athanassiou, A. All-natural composite wound dressing films of essential oils encapsulated in sodium alginate with antimicrobial properties. Int. J. Pharm. 2013, doi:10.1016/j.ijpharm.2013.10.046.

11. Dalmoro, A.; Barba, A.; Lamberti, G.; d’Amore, M. Intensifying the microencapsulation process: Ultrasonic atomization as an innovative approach. Eur. J. Pharm. Biopharm. 2012, 80, 471-477.

12. Dalmoro, A.; d’Amore, M.; Barba, A. Droplet size prediction in the production of drug delivery microsystems by ultrasonic atomization. Transl. Med. 2013, 7, 6-11.

13. Chan, E.-S.; Lee, B.-B.; Ravindra, P.; Poncelet, D. Prediction models for shape and size of Ca-alginate macrobeads produced through extrusion-dripping method. J. Colloid Interface Sci. 2009, 338, 63-72.

14. Quong, D.; Neufeld, R.J.; Skjak-Braek, G.; Poncelet, D. External versus internal source of calcium during the gelation of alginate beads for DNA encapsulation. Biotechnol. Bioeng. 1998, 57, 438-446.

15. Reis, C.P.; Ribeiro, A.J.; Veiga, F.; Neufeld, R.J.; Damgé, C. Polyelectrolyte biomaterial interactions provide nanoparticulate carrier for oral insulin delivery. Drug Deliv. 2008, 15, 127-139.

16. Falé, P.L.; Borges, C.; Madeira, J.P.A.; Ascensão, L.; Araújo, M.E.M.; Florêncio, M.H.; Serralheiro, M.L.M. Rosmarinic acid, scutellarein 4'-methyl ether 7-O-glucuronide and (16S)-coleon E are the main compounds responsible for the antiacetylcholinesterase and antioxidant activity in herbal tea of Plectranthus barbatus ("falso boldo"). Food Chem. 2009, 114, 798-805.

17. Roberto, A.; Caetano, P.P. A high-throughput screening method for general cytotoxicity part I chemical toxicity. Revista Lusófona de Ciências e Tecnologias de Saúde 2005, 2, 95-100.

18. Fernandes, A.S.; Gaspar, J.; Cabral, M.F.; Rueff, J.; Castro, M.; Batinic-Haberle, I.; Costa, J.; Oliveira, N.G. Protective role of ortho-substituted Mn(III) $N$-alkylpyridylporphyrins against the oxidative injury induced by tert-butylhydroperoxide. Free Radic. Res. 2010, 44, 430-440.

19. Ostberg, L.; Graffner, C. Calcium alginate matrices for oral multiple unit administration: II. Effect of process and formulation factors on matrix properties. Int. J. Pharm. 1993, 97, 183-193.

20. Braccini, I.; Pérez, S. Molecular basis of $\mathrm{C}(2+)$-induced gelation in alginates and pectins: The egg-box model revisited. Biomacromolecules 2001, 2, 1089-1096. 
21. Gray, C.J.; Dowsett, J. Retention of insulin in alginate gel beads. Biotechnol. Bioeng. 1988, 31, 607-612.

22. Chen, L.; Subirade, M. Effect of preparation conditions on the nutrient release properties of alginate-whey protein granular microspheres. Eur. J. Pharm. Biopharm. 2007, 65, 354-362.

23. Reis, C.; Veiga, F.; Ribeiro, A.J.; Neufeld, R.J.; Damgé, C. Nanoparticulate biopolymers deliver insulin orally eliciting pharmacological response. J. Pharm. Sci. 2008, 97, 5290-5305.

(C) 2014 by the authors; licensee MDPI, Basel, Switzerland. This article is an open access article distributed under the terms and conditions of the Creative Commons Attribution license (http://creativecommons.org/licenses/by/3.0/). 